\title{
Liver morbidity due to Schistosoma mekongi in Cambodia after seven rounds of mass drug administration
}

\author{
H. Keang ${ }^{a, b}$, P. Odermatt ${ }^{b, c, *}$, S. Odermatt-Biays ${ }^{b}$, S. Cheam ${ }^{a}$, \\ A. Degrémont ${ }^{b}, C$. Hatz ${ }^{c}$
}

\author{
a Provincial Hospital of Kratie, Kratie, Cambodia \\ ${ }^{\mathrm{b}}$ Institut de la Francophonie pour la médecine tropicale, Vientiane, Lao PDR \\ c Swiss Tropical Institute, Postfach, 4002 Basel, Switzerland
}

Received 31 October 2006; received in revised form 6 April 2007; accepted 11 April 2007

\section{KEYWORDS \\ Schistosoma mekongi; Liver disease; Pathology; Morbidity; Evaluation; Ultrasonography}

\begin{abstract}
Summary Severe liver disease due to Schistosoma mekongi was frequent in northern Cambodia. Between 1995 and 2002, seven rounds of mass chemotherapy (praziquantel) reduced infection from $50 \%$ to below $3 \%$. In 2002, we assessed hepatosplenic morbidity by historical, clinical and ultrasonographic investigations in adults (older than 14 years) from endemic $(n=342)$ and non-endemic $(n=103)$ areas (Kratie province). Clinical hepatomegaly ( $25 \mathrm{vs.} 0 \%)$, splenomegaly ( 55 vs. $0 \%$ ), reported blood in stool (41 vs. $20 \%$ ) and abdominal pain (78 vs. $57 \%$ ) were significantly higher in the endemic area. In this area, significantly more subjects reported a family history of death due to schistosomiasis (12 vs. 0\%); 63\% (vs. 0\%) reported having at least three treatments of praziquantel in previous years; and only $11 \%$ (vs. 99\%) had normal liver ultrasonographic examination. Periportal fibrosis with portal hypertension was diagnosed in $46 \%$ (vs. $0 \%$ ) of people in this area; $18 \%$ (vs. $0 \%$ ) and $5 \%$ (vs. $0 \%$ ) of portal hypertension was classified as moderate and severe, respectively. People aged between 24 and 35 years were mostly affected. There was no gender difference. The pathology in the endemic district is most probably residual morbidity of $S$. mekongi infections. Contributions of co-infections (hepatitis) cannot be excluded. Careful monitoring of the affected communities is required.

(c) 2007 Royal Society of Tropical Medicine and Hygiene. Published by Elsevier Ltd. All rights reserved.
\end{abstract}

* Corresponding author. Tel.: +41 6128482 14; fax: +41612848105.

E-mail address: peter.odermatt@unibas.ch (P. Odermatt).

\section{Introduction}

Schistosoma mekongi infection may lead to severe intestinal, hepatosplenic and cerebral morbidity. Mortality due to this parasite was shown to be dramatic in non-treated transmission areas (Biays et al., 1999; Urbani et al., 2002), occurring mostly through oesophageal bleeding. Recently, a 
case of cerebral infection has been described (Houston et al., 2004).

In 1968, the first cases of S. mekongi originating from Kratie, Cambodia, were diagnosed (Audebaud et al., 1968; Tournier-Lasserve et al., 1970). In the subsequent period of war and political instability, follow-up field investigations in the province of Kratie were not possible. Ongoing transmission led to a high degree of morbidity and mortality in the districts of Sambo and Kratie, situated in the northern part of Kratie province.

In 1994, approximately 10 patients with severe schistosomiasis were hospitalized each month in the district hospital of Sambo and more than 150 affected patients were treated in the outpatient clinic (Biays et al., 1999). The clinical picture of the severe cases included advanced hepatosplenomegaly, ascites and other signs of portal hypertension. Death due to spleen rupture or oesophageal bleeding was frequently observed in Sambo District Hospital and Kratie Provincial Hospital and was often reported from the communities.

In 1995, the entire population of three villages of Sambo district (Samerong, Chatnaol and Achen) was examined. Forty-nine per cent were clinically diagnosed with hepatomegaly, reaching as high as 90\% in children aged 10-14 years and 60\% in young adults aged $15-19$ years (Stich et al., 1999). In addition, in a sample from 20 primary schools in the districts of Sambo and Kratie, the prevalence of infection and hepatomegaly in school children reached 70 and $90 \%$, respectively (Stich et al., 1999).

In the same year, a control programme based on annual chemotherapy was started. The intervention targeted the schistosomiasis-endemic districts of Sambo and Kratie populated by approximately 60000 people. Up to 2002, seven annual mass drug administration campaigns were carried out in which all individuals (except children $<2$ years and pregnant women) were treated with praziquantel $(40 \mathrm{mg} / \mathrm{kg}$ body weight) in combination with mebendazole $(500 \mathrm{mg})$. The annual coverage of treatment was between 62 and $74 \%$ (Sinuon et al., 2007).

Over the control programme period, between 1995 and 2002, monitoring surveys showed that liver and intestinal morbidity was reduced. Clinical manifestations, such as hepatomegaly and splenomegaly, and/or reports of blood and mucus in stool have been drastically reduced in schoolchildren in four sentinel villages, between 1995 and 2002, as have the numbers of schistosomiasis patients diag- nosed in the health facilities in the endemic area (Urbani et al., 2002). The infection rates were monitored annually by stool analysis of schoolchildren in four sentinel villages. The data showed a marked decline in infection from more than $60 \%$ to less than $3 \%$ (Ohmae et al., 2004). Health promotion activities and the provision of safe water sources led to general improvements in the target communities. Overall, the intervention was very successful with regard to infection and severe morbidity control (Sinuon et al., 2007).

Ultrasonography is used to assess liver morbidity due to Schistosoma infection (Richter et al., 2003). Guidelines for standardised ultrasonographic assessment are available (WHO, 2000) and allow the evaluation of large-scale control programmes (Hatz, 2001). Periportal fibrosis and portal hypertension are the primary indicators for the disease. Thus ultrasonography has been used to assess $S$. japonicum foci (Hatz et al., 1992), but only very few data are available for S. mekongi-endemic areas.

The objective of the present study conducted in 2002 was to measure the prevalence of liver morbidity attributable to S. mekongi in the endemic area of Sambo district following seven rounds of annual mass drug administration, by comparing it with signs, symptoms and measures of pathology in the non-endemic untreated area of Chhlong district. The study was conducted using family histories, clinical examinations and ultrasonographies.

\section{Materials and methods}

The study was carried out in the province of Kratie in May and June 2002. A cross-sectional sample survey was conducted in four schistosomiasis-endemic villages of Ampil Teuk, Kampong Krabei, Tongsaong Thleak and Sambo (Table 1) in the district of Sambo (district population: 39 402 habitants in 2002), in which in 1994, during a baseline survey prior to control activities, a high prevalence of liver morbidity was found (Stich et al., 1999). Households were identified by random selection from the list of households provided by the village chief. All subjects older than 14 years and present on the survey day were enrolled.

Chryothmar village (Table 1), located on the shore of the Mekong and capital of the Chhlong district (district population in 2002: 47919 habitants), was chosen as a comparison. Enrolment procedures were the same as in the Sambo district.

Table 1 Population and study sample

\begin{tabular}{|c|c|c|c|c|c|c|}
\hline \multirow[t]{2}{*}{ District } & \multirow[t]{2}{*}{ Village } & \multirow[t]{2}{*}{ Population } & \multicolumn{4}{|c|}{ Sample } \\
\hline & & & $n$ & $\%$ female & Age range (years) & Mean age (years) \\
\hline \multirow[t]{4}{*}{ Sambo } & Tongsaong Thleak & 1634 & 72 & 68.1 & $15-69$ & 34.8 \\
\hline & Kampong Krabei & 662 & 94 & 62.8 & $14-55$ & 31.2 \\
\hline & Ampil Teuk & 1063 & 96 & 58.3 & $15-53$ & 29.1 \\
\hline & Sambo & 2245 & 80 & 60.0 & $15-55$ & 29.3 \\
\hline Subtotal & & 5604 & 342 & 62.0 & $14-69$ & 30.9 \\
\hline Chhlong & Chryothmar & 3614 & 103 & 56.3 & $16-63$ & 37.0 \\
\hline Total & & 9218 & 445 & 60.7 & $14-69$ & 32.3 \\
\hline
\end{tabular}


Chhlong district in the southern part of Kratie province is only marginally affected by S. mekongi transmission. It is considered a non-endemic area. The river ecology is profoundly different. Sandy shores prevail and are unfavourable for the development of the intermediate snail host Neotricula aperta, which requires rocky underground material (Attwood et al., 2004; Ohmae et al., 2004). Schistosoma mekongi infections have rarely been reported in this district and a sero-epidemiological survey, using S. japonicum antigens, showed that fewer than $20 \%$ of the schoolchildren tested positive (Ohmae et al., 2004). In contrast, the districts of Sambo and Kratie had a seroprevalence of more than $95 \%$. Consequently, no treatment campaigns were performed in Chhlong district.

All enrolled people were interviewed for a history of haematemesis, bloody stool, abdominal pain and diarrhoea in the past 2 weeks, family history of deaths due to schistosomiasis and the number of treatments with praziquantel. Subsequently, each subject underwent a directed clinical examination for the presence of hepatomegaly, splenomegaly and shunt circulation, ascites and jaundice. Hepatomegaly was scored by palpating the left liver lobe along the xiphoid-umbilicus line in supine position. Hepatomegaly was defined as absent when the left liver lobe was not palpable and as present when the left liver lobe was palpable under the xiphoid. The presence of ascites was assessed by percussion and fluid thrill. Spleen enlargement was registered using the Hackett score (Hackett, 1944).

Liver pathology was assessed with an ultrasonographic examination following the Niamey protocol (WHO, 2000) by using a Fukuda Denshi UF-4000 (scan sectorial FUTC111A 3,5 MHz 60R convex probe) portable ultrasonography machine. We assessed the score of image patterns of the liver parenchyma (IP score), the height-standardised score for periportal thickening (PT score) and periportal hypertension ( $\mathrm{PH}$ score). $\mathrm{PH}$ score consisted of the sum of the height-standardised portal vein diameter (portal vein score: 0,4 or 6 ), the presence of collateral veins (collateral score: 0 or 4 ) and the presence of ascites (ascites score: 0 or 3 ). The final score consisted of the IP score, PT score and the $\mathrm{PH}$ score. Liver surface, shape of the caudal liver edge and height-standardised lengths of the right and left liver lobes were assessed but reported independently of the scoring results.

We aimed to enrol 82 adults per village, which allowed for estimating a factor of unknown prevalence $(P=0.5)$ with a precision of 0.15 (power $80 \%$, alpha 0.05 ) at the village level and 0.08 for all the four villages in the S. mekongiendemic area $(n=328)$. All data were entered into Epilnfo version 6.04 (CDC, Atlanta, GA, USA). Data were analysed with STATA version 8.2 (Stata Corp., College Station, TX, USA). Excess risk was calculated for the $S$. mekongi-endemic area by subtracting prevalence of absolute risks from the endemic area minus the non-endemic area and exact $95 \%$ confidence intervals were calculated ( $95 \% \mathrm{CI}$ ER). For data on the portal hypertension score and the final score of the endemic district, an ordered logistic regression was used to test whether age groups and gender independently contribute to the observed morbidity pattern. The data were grouped in four age groups: 14-24 years, 25-34 years, 35-44 years and 45 years and older.
The study followed the ethical conduct defined in the guidelines of the Declaration of Helsinki (1964). All patients gave their informed oral consent before being included in the study.

\section{Results}

A total of 445 people was enrolled in the study (Sambo district 342, Chhlong district 103). Details of the sample are given in Table 1. In Sambo district 34.2, 33.3, 18.7, 13.7 and $1.5 \%$ fell into the $14-24,25-34,35-44$ and 45 years and older age group, respectively, while in Chhlong district $18.5,23.3,29.1$ and $29.1 \%$ of the subjects were in the respective age groups. The median age in Sambo district was 30 years, significantly younger than in Chhlong district (37 years, $P<0.001$ ). There was no difference in mean age between the sexes, or sex ratio between the two districts $(P=0.3)$.

The socio-economic status of the examined population of Sambo was significantly lower than Chhlong district. A significantly smaller proportion of the study participants in Sambo district attended secondary school (14.6 vs. $33.0 \%$, $P<0.001$, controlled for age group). Most of the studied Sambo population were rice farmers, whereas this activity was rare in the studied village of Chhlong district (79.8 vs. 9.7\%).

Clinical examination revealed schistosomiasis-related morbidity in Sambo district (Table 2). Hepatomegaly (24.3\%), splenomegaly (54.7\%) and clinical signs of portal hypertension such as ascites (1.2\%) and collateral veins (2.0\%) were seen only in people in the schistosomiasisendemic district. Jaundice was diagnosed in three patients (1.2\%). Blood in stool and abdominal pain was reported significantly more often in Sambo than in Chhlong district $(P<0.001)$, while no difference was recorded for diarrhoea.

Twelve per cent of people in the district of Sambo reported to have lost at least one family member due to schistosomiasis in recent years compared with no reports in the non-endemic district of Chhlong.

In Sambo district, at least three treatments with praziquantel during mass drug administration were reported by $62.5 \%$ of the people and $21.9 \%$ reported to have been treated six or seven times. No treatment reports were recorded in Chhlong district.

Ultrasonographic investigations revealed major liver pathology in the schistosomiasis-endemic district of Sambo, while very little pathology was found in Chhlong district (Table 3 ). In almost half the examined subjects (45.5\%), we diagnosed advanced periportal fibrosis with portal hypertension. In Sambo district, more than one-fifth of the subjects $(23.1 \%)$ had moderate or severe portal hypertension (PH score) and periportal vein thickening was prevalent. In Sambo, $40 \%$ of subjects showed highly echogenic ruff areas around the portal bifurcation and the main stems of the liver parenchyma. In addition, we frequently diagnosed severe fibrotic liver parenchyma, consisting of high echogenic patches extending from the main portal vein and branches into the parenchyma ( $23 \%$ of subjects). No reticular patterns of liver morbidity were identified.

Analysis of the liver morbidity scores in Sambo district revealed a similar pattern in all villages and in both 
Table 2 Results of the history and clinical examination of 445 study participants

\begin{tabular}{|c|c|c|c|c|}
\hline & \multirow[t]{2}{*}{ Sambo $(n=342) \%$} & \multirow[t]{2}{*}{ Chhlong $(n=103) \%$} & \multicolumn{2}{|c|}{ Excess risk in endemic area } \\
\hline & & & $\%$ & $95 \% \mathrm{Cl}$ \\
\hline \multicolumn{5}{|l|}{ History } \\
\hline Haematemesis & 5.9 & 1.0 & 4.8 & $1.8-8.0$ \\
\hline Blood in stool (past 2 weeks) ${ }^{a}$ & 40.6 & 20.4 & 20.2 & $10.9-29.6$ \\
\hline Abdominal pain (past 2 weeks) & 77.5 & 57.3 & 20.2 & $9.7-30.7$ \\
\hline Diarrhoea (past 2 weeks) ${ }^{\mathrm{b}}$ & 44.4 & 35.9 & 8.5 & $2.1-19.2$ \\
\hline \multicolumn{5}{|l|}{ Family death due to schistosomiasis } \\
\hline None & 88.0 & 100 & - & - \\
\hline 1 person & 7.6 & 0 & 7.6 & $4.8-10.4$ \\
\hline 2 or 3 people & 4.4 & 0 & 4 & $2.2-6.6$ \\
\hline \multicolumn{5}{|l|}{ Praziquantel treatment } \\
\hline 0 & 7.0 & 100 & - & - \\
\hline $1-2$ times & 30.4 & 0 & 30.4 & $25.5-35.3$ \\
\hline $3-5$ times & 40.6 & 0 & 40.6 & $35.4-45.8$ \\
\hline $6-7$ times & 21.9 & 0 & 21.9 & $17.5-26.3$ \\
\hline \multicolumn{5}{|l|}{ Clinical examination } \\
\hline Hepatomegaly (present) & 24.3 & 0 & - & $19.7-28.8$ \\
\hline \multicolumn{5}{|l|}{ Splenomegaly (Hackett score) ${ }^{c}$} \\
\hline 0 & 45.3 & 100 & - & - \\
\hline 1 & 12.6 & 0 & 12.6 & $9.1-16.1$ \\
\hline $2-3$ & 32.1 & 0 & 32.1 & $27.2-37.1$ \\
\hline $4-5$ & 9.9 & 0 & 9.9 & $6.8-13.1$ \\
\hline Ascites (present) & 1.2 & 0 & 1.2 & $0.03-2.3$ \\
\hline Collateral veins (present) & 2.0 & 0 & 2.0 & $0.5-3.5$ \\
\hline Jaundice (present) & 1.2 & 0 & 1.2 & $0.03-2.3$ \\
\hline
\end{tabular}
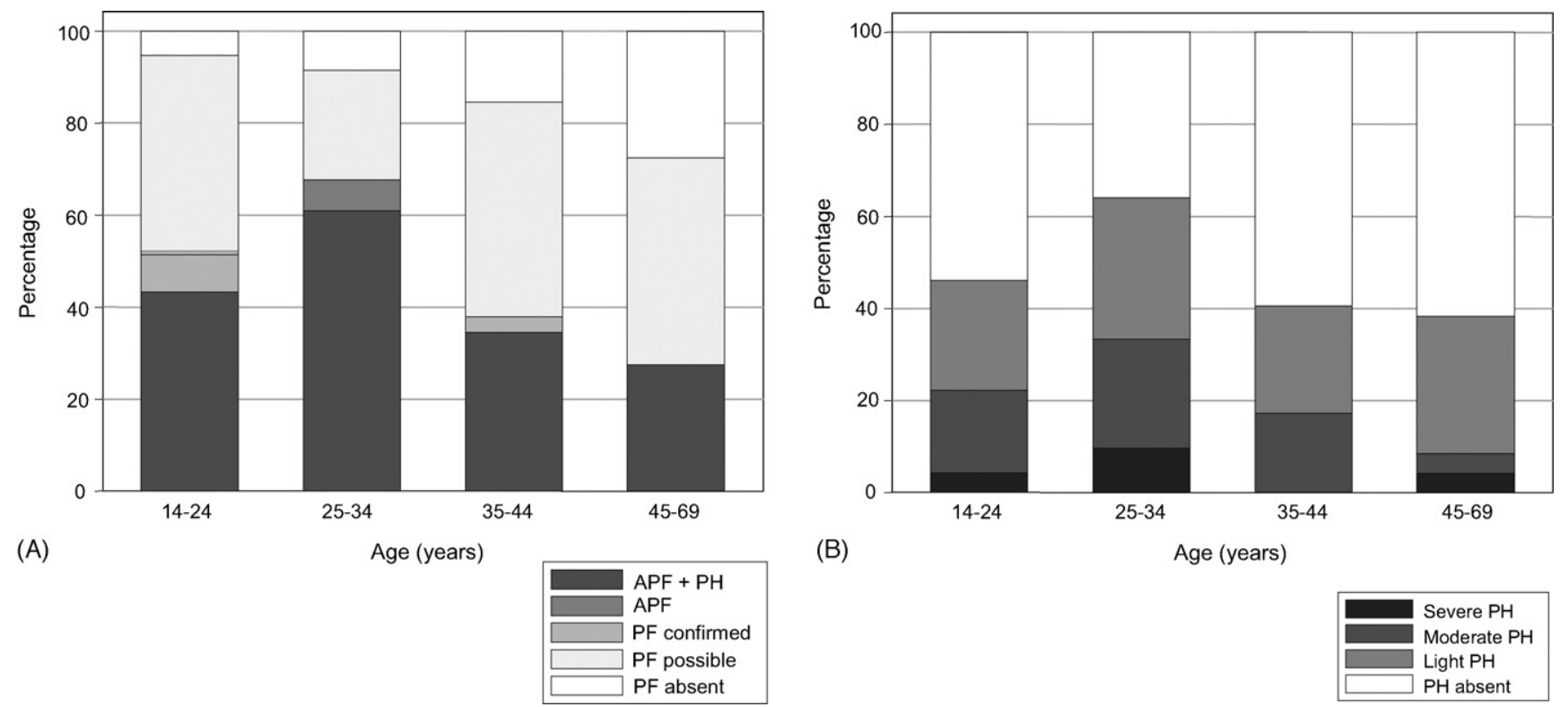

(B)

Age (years)

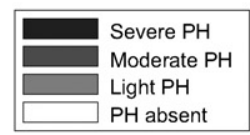

Figure 1 (A) Final score of ultrasonography assessment by age in Sambo district ( $n=342$; PF: periportal fibrosis; APF: advanced PF; PH: portal hypertension). (B) Portal hypertension (PH) score by age in schistosomiasis-endemic district of Sambo $(n=342)$. 
Table 3 Results of ultrasonographic examinations in Sambo and Chhlong districts

\begin{tabular}{|c|c|c|c|c|}
\hline & \multirow[t]{2}{*}{ Sambo $(n=342) \%$} & \multirow[t]{2}{*}{ Chhlong $(n=103) \%$} & \multicolumn{2}{|c|}{ Excess risk in endemic area } \\
\hline & & & $\%$ & $95 \% \mathrm{Cl}$ \\
\hline \multicolumn{5}{|l|}{ Final score ${ }^{a}$} \\
\hline No PF & 11.2 & 99.0 & - & \\
\hline PF possible & 37.3 & 1.0 & 36.2 & $30.5-40.9$ \\
\hline PF confirmed & 1.0 & 0 & 1.0 & $0.1-2.0$ \\
\hline $\mathrm{APF}$ & 5.1 & 0 & 5.1 & $2.7-7.5$ \\
\hline APF + portal hypertension & 45.5 & 0 & 45.5 & $40.0-50.0$ \\
\hline \multicolumn{5}{|l|}{ PH score (grouped) } \\
\hline 0 normal & 50.0 & 95.1 & - & - \\
\hline 4 light & 26.9 & 4.9 & 22.0 & $15.8-28.3$ \\
\hline $6-8$ moderate & 17.8 & 0 & 17.8 & $13.8-21.9$ \\
\hline $10-13$ severe & 5.3 & 0 & 5.3 & $2.9-7.6$ \\
\hline \multicolumn{5}{|l|}{ PT score } \\
\hline 0 normal & 0.9 & 1.9 & - & - \\
\hline 1 slightly increased & 86.6 & 98.1 & - & - \\
\hline 4 increased & 10.2 & 0 & 10.2 & $7.0-13.4$ \\
\hline 8 much increased & 2.3 & 0 & 2.3 & $0.7-3.9$ \\
\hline \multicolumn{5}{|l|}{ IP score ${ }^{b}$} \\
\hline 0 & 0.9 & 1.9 & - & - \\
\hline 1 & 10.8 & 97.1 & - & - \\
\hline 2 & 24.6 & 1.0 & 23.6 & $18.7-28.5$ \\
\hline 4 & 39.5 & 0 & 39.5 & $34.3-44.7$ \\
\hline 6 & 22.5 & 0 & 22.5 & $18.1-26.9$ \\
\hline 8 & 1.8 & 0 & 1.8 & $0.4-3.1$ \\
\hline \multicolumn{5}{|l|}{ Liver surface (frontal) } \\
\hline Regular & 6.4 & 98.1 & - & - \\
\hline Lightly nodular & 74.0 & 1.9 & 72.0 & $66.7-77.4$ \\
\hline Nodular & 19.6 & 0 & 19.6 & $15.4-23.8$ \\
\hline
\end{tabular}

PF: periportal fibrosis; APF: advanced PF; PH: portal hypertension; PT: periportal vein thickening; IP: image pattern.

a $n=412$ ( $92.6 \%$ of total); for 33 patients final score could not be established.

b Short description of IP score: 0, normal; 1, diffuse foci; 2, ring echoes; 4, ruff zones around portal bifurcation and main stem; 6, patches extending from main portal vein and branches to parenchyma; 8 , bands and streaks extending from main portal vein and its bifurcation to liver surface.

sexes. However, substantial age differences were observed. The population between 25 and 34 years of age was mostly affected. Of them, 61.0 and $64.4 \%$ suffered from advanced periportal fibrosis with portal hypertension and portal hypertension, respectively, while in other age groups corresponding rates were much lower (Figure 1).

Ordered multiple regression analysis showed that age group rather than gender contributes to the advanced periportal fibrosis and portal hypertension. Subjects aged 25 to 34 had a 1.75-fold risk of an increased final score compared with the youngest age group, controlled for sex and age group $(95 \% \mathrm{Cl} 1.04-2.94, P=0.033)$. Similar results were obtained for the portal hypertension score. Subjects aged 25 to 34 years had double the risk for portal hypertension compared with the youngest age group, controlled for sex and age group $(\mathrm{OR}=2.02,95 \% \mathrm{Cl} 1.24-3.29, P=0.005)$. The risks for portal hypertension for the older age groups were not significantly different from those of the 14-24 year olds $(\mathrm{OR}=0.76,95 \% \mathrm{Cl} 0.42-1.38, P=0.368$ and $\mathrm{OR}=0.64,95 \%$ $\mathrm{Cl} 0.33-1.24, P=0.187$ for subjects aged $35-44$ and $45-69$ years, respectively).

\section{Discussion}

Our study reveals a high degree of sub-clinical liver morbidity, reaching a prevalence of more than $60 \%$ of advanced periportal fibrosis and portal hypertension in young adults (aged 25-34 years) in the district of Sambo, Kratié Province, Cambodia. Younger and older age groups were also affected, but substantially less so. Furthermore, very little liver pathology could be detected in the district of Chhlong, suggesting that the observed morbidity in Sambo district was an excess morbidity attributable to $S$. mekongi infections.

In 1995, in Sambo district, left liver lobe enlargement was clinically observed in high frequencies in the communities (Biays et al., 1999). In the hospitals of this province these observations were confirmed. Furthermore, the consequences of severe portal hypertension, such as ascites and oesophageal bleeding, were frequent and led to a great number of casualties. In 1998, 106 chronic patients with portal hypertension were identified. Eleven were eligible for surgical decompression procedures in order to decrease portal hypertension (Dumurgier et al., 2006). 
Histological liver examinations of six of these patients revealed a surprisingly high degree of fibrosis (Monchy et al., 2006). No similar observations were made in the Chhlong district.

Sub-clinical liver morbidity could not be assessed in 1995. Therefore, no data are available for a direct comparison with our study. However, an ulrasonographic assessment was performed in 1997 in a S. mekongi-endemic village in the Stung Treng province (north of Kratie province). Of 223 subjects, $73 \%$ were found positive for S. mekongi, and periportal thickening and/or portal vein enlargement was diagnosed in $84 \%$ (Urbani et al., 2002). Therefore, it is reasonable to assume that sub-clinical morbidity was present in comparable frequencies for 1995 in the Kratie province.

Undoubtedly, between 1994 and 2002, the situation has much improved in terms of infection and morbidity (Sinuon et al., 2007). The prevalence of clinically observed hepatomegaly has dropped from over $60 \%$ to an undetectable level in annually examined sentinel primary schools (Ohmae et al., 2004). Our study diagnosed hepatomegaly in $25 \%$ of our subjects. In 1994, in comparable age groups, more than $80 \%$ had the same finding.

Based on the high number of mass drug administrations we assumed that most S. mekongi infections were cured. Consequently, the question arises whether the observed subclinical liver morbidity is indeed a residual part of the $S$. mekongi infection-induced pathology in the population, or provoked by other factors such as hepatitis B (HBV) and $C$ (HCV) infections. Hepatitis infections may lead to hepatic reticular lesions of similar ultrasonographic appearance (Kardorff et al., 1999). As a community survey in 1991 indicated that $8.0 \%$ of the Cambodian population was hepatitis B surface antigen positive and $6.5 \%$ was anti-HCV positive (Thuring et al., 1993), these infections could have potentially contributed to our morbidity findings. Furthermore, Cambodia has one of the world's highest overall injection usages (Vong et al., 2005), suggesting a high rate of overuse and unsafe injection practices, which may lead to these infections. Therefore, a contribution of hepatitis viruses to the observed liver morbidity cannot be excluded. However, we believe that a major part of this liver pathology is attributable to $S$. mekongi infection, for the following reasons.

Firstly, we observed liver pathology in a high prevalence of $46 \%$. In 1998, in an attempt to select chronic schistosomiasis patients for surgical treatment, 106 severe schistosomiasis cases were examined, $30 \%$ of whom were found positive for HBV or HCV antigen (19\% HBV, 9\% HCV, 2\% both; Dr Muth Sinuon, National Malaria Centre, Phnom Penh, personal communication). In a sample of 421 individuals in Kratie province, Chigusa and colleagues found $20.2 \%$ positive HBV (Chigusa et al., 2006). Such a prevalence could be expected in our study population. But most probably it was lower because subjects in our study were not sick and therefore had less frequent contact with a health facility compared with the chronic severe cases of schistosomiasis. Hence, our study population was at lower risk for hepatitis infection.

Secondly, hepatitis infections provoke hepatic reticular lesions, mainly at the late stage of the disease. Therefore, one would expect to find liver pathology in older individuals. However, in our study we found pathology predominantly in young adults (aged 25-34 years) and adolescents (aged 14-24 years).

Thirdly, a 2-year cohort study of liver morbidity due to S. japonicum showed that after a single treatment with praziquantel, sub-clinical liver morbidity was significantly reduced (Li et al., 2000), but even after 5 years followup the resolution was not complete (Li et al., 2002). It was concluded that pathological changes around the portal tree might regress to a large degree but that 'network pattern' abnormalities do not normally resolve (Richter, 2000). A comparable finding was obtained in a 2-year cohort study in Stung Treng province in Cambodia for S. mekongi between 1997 and 1999 (Hatz, 2001). While at the start of the cohort only $18.1 \%$ of subjects had a normal liver ultrasonographic assessment (pattern A or B of Niamey protocol), after 2 years $81.6 \%$ did not reveal pathological liver lesions (Urbani et al., 2002). But, in all patients, the resolution of liver morbidity was incomplete. Based on these findings, liver pathology is to be expected in the S. mekongi-endemic areas.

Finally, very little pathological finding was found in Chhlong district, where S. mekongi is rare. We would expect that with a high rate of injection drug use, and a resulting high risk for hepatitis, at least some hepatic lesions should have been found. This, however, was not the case.

A surprising finding was that a substantial number of subjects in Chhlong district reported blood in stool. Serological studies showed that some infections with S. mekongi were present in this district, which explains these reports to some extent (Ohmae et al., 2004). In addition, other infections, such as by Strongyloides spp., might have contributed to the observation. Furthermore, mis-reporting cannot be excluded.

Our study followed the recommendations on the morbidity assessment procedures for Asian schistosomiasis established by WHO expert committee in Phnom Penh (Cambodia, 2002) and Yueyang (Hunan Province, China, 2005) (WHO, 2007). The study suffers from epidemiological weaknesses. The sampling procedures resulted in a female-dominated sample population, as many men were away in the rice fields. The sample was not proportional to the population size, and no information could be obtained from non-participating individuals. Furthermore, the districts could not be matched for other prognostic variables. These factors limit the strength of the conclusions that can be drawn about the general population. In addition, due to lack of resources we were not able to perform laboratory investigations such as stool examinations for S. mekongi and serological analysis for hepatitis virus infection.

Nonetheless, our study demonstrates that a substantial amount of sub-clinical morbidity is present in a schistosomiasis-endemic district, which was probably to a large extent residual morbidity due to $\mathrm{S}$. mekongi. The strength of our study is that we included a control group from a non-endemic district and demonstrated the amount of sub-clinical morbidity in a community where little active S. mekongi infection is present. It furthermore showed that gross clinical morbidity was rare, providing further evidence for a successful intervention. Careful follow-up and monitoring are required, and necessary action needs to be taken to maintain and consolidate gains from preceding control efforts. 
Authors' contributions: PO, HK, SOB and $\mathrm{CH}$ designed the study protocol; HK carried out assessments under the supervision of $\mathrm{PO}$ and $\mathrm{SC} ; \mathrm{HK}, \mathrm{PO}$ and $\mathrm{AD}$ analysed and interpreted the data; HK wrote the draft manuscript. All authors contributed to and approved the final version. $\mathrm{PO}, \mathrm{CH}$ and $\mathrm{AD}$ are guarantors of the paper.

Acknowledgements: The participation of the authorities and the population in the selected villages is highly acknowledged. Many thanks to Prof. T.A. Smith for statistical support, $\mathrm{Dr}$ T. Mak for commenting on a draft manuscript and Mr D. Anderegg for improving the English.

Funding: Agence Universitaire de la Francophonie provided a scholarship to $\mathrm{Dr} \mathrm{H}$. Keang and funded this study.

Conflicts of interest: None declared.

Ethical approval: Ministry of Health and the provincial health department of Kratie province, Cambodia.

\section{References}

Attwood, S.W., Campbell, I., Upatham, E.S., Rollinson, D., 2004. Schistosomes in the Xe Kong river of Cambodia: the detection of Schistosoma mekongi in a natural population of snails and observations on the intermediate host's distribution. Ann. Trop. Med. Parasitol. 98, 221-230.

Audebaud, G., Tournier-Lasserve, C., Brumpt, V., Jolly, M., Mazaud, R., Imbert, X., Bazillio, R., 1968. 1st case of human schistosomiasis observed in Cambodia (Kratie area). Bull. Soc. Pathol. Exot. Filiales. 61, 778-784 [in French].

Biays, S., Stich, A.H., Odermatt, P., Long, C., Yersin, C., Men, C., Saem, C., Lormand, J.D., 1999. A foci of Schistosomiasis mekongi rediscovered in Northeast Cambodia: cultural perception of the illness; description and clinical observation of 20 severe cases. Trop. Med. Int. Health 4, 662-673 [in French].

Chigusa, Y., Ohmae, H., Otake, H., Keang, H., Sinuon, M., Saem, C., Socheat, D., Matsuda, H., 2006. Effects of repeated praziquantel treatment on schistosomiasis mekongi morbidity as detected by ultrasonography. Parasitol. Int. 55, 261-265.

Dumurgier, C., Tay, K.H., Surith, T.N., Rathat, C., Buisson, Y., Monchy, D., Sinuon, M., Socheat, D., Urbani, C., Chaem, S., Huerre, M., Kheang, H., 2006. Place of surgery in the prevention of recurrences of digestive haemorrhages at the patients presenting a portal hypertension due to Schistosoma mekongi. Bull. Soc. Pathol. Exot. 99, 365-371 [in French].

Hackett, L., 1944. Spleen measurement in malaria. J. Natl. Malar. Soc. 3, 121-123.

Hatz, C.F., 2001. The use of ultrasound in schistosomiasis. Adv. Parasitol. 48, 225-284.

Hatz, C., Murakami, H., Jenkins, J.M., 1992. A review of the literature on the use of ultrasonography in schistosomiasis with special reference to its use in field studies. 3. Schistosoma japonicum. Acta Trop. 51, 29-36.

Houston, S., Kowalewska-Grochowska, K., Naik, S., McKean, J., Johnson, E.S., Warren, K., 2004. First report of Schistosoma mekongi infection with brain involvement. Clin. Infect. Dis. 38, e1-e6.
Kardorff, R., Olveda, R.M., Acosta, L.P., Duebbelde, U.J., Aligui, G.D., Alcorn, N.J., Doehring, E., 1999. Hepatosplenic morbidity in schistosomiasis japonica: evaluation with Doppler sonography. Am. J. Trop. Med. Hyg. 60, 954-959.

Li, Y.S., Sleigh, A.C., Ross, A.G., Li, Y., Williams, G.M., Tanner, M., McManus, D.P., 2000. Two-year impact of praziquantel treatment for Schistosoma japonicum infection in China: re-infection, subclinical disease and fibrosis marker measurements. Trans. R. Soc. Trop. Med. Hyg. 94, 191-197.

Li, Y.S., Sleigh, A.C., Li, Y., Tanner, M., Dessein, A., Williams, G.M., McManus, D.P., 2002. Five-year impact of repeated praziquantel treatment on subclinical morbidity due to Schistosoma japonicum in China. Trans. R. Soc. Trop. Med. Hyg. 96, 438-443.

Monchy, D., Dumurgier, C., Heng, T.K., Hong, K., Khun, H., Hou, S.V., Sok, K.E., Huerre, M.R., 2006. Histology of liver lesions due to Schistosoma mekongi. About six cases with severe portal hypertension operated in Cambodia. Bull. Soc. Pathol. Exot. 99 359-364 [in French].

Ohmae, H., Sinuon, M., Kirinoki, M., Matsumoto, J., Chigusa, Y., Socheat, D., Matsuda, H., 2004. Schistosomiasis mekongi: from discovery to control. Parasitol. Int. 53, 135-142.

Richter, J., 2000. Evolution of schistosomiasis-induced pathology after therapy and interruption of exposure to schistosomes: a review of ultrasonographic studies. Acta Trop. 77, 111-131.

Richter, J., Hatz, C., Haussinger, D., 2003. Ultrasound in tropical and parasitic diseases. Lancet 362, 900-902.

Sinuon, M., Tsuyuoka, R., Socheat, D., Odermatt, P., Ohmae, H., Matsuda, H., Montresor, A., Paris, J.C., 2007. Control of Schistosoma mekongi in Cambodia: results of eight years of control activities in the two endemic provinces. Trans. R. Soc. Trop. Med. Hyg. 101, 34-39.

Stich, A.H., Biays, S., Odermatt, P., Men, C., Saem, C., Sokha, K., Ly, C.S., Legros, P., Philips, M., Lormand, J.D., Tanner, M., 1999. Foci of Schistosomiasis mekongi, Northern Cambodia: II. Distribution of infection and morbidity. Trop. Med. Int. Health 4 674-685.

Thuring, E.G., Joller-Jemelka, H.I., Sareth, H., Sokhan, U., Reth, C., Grob, P., 1993. Prevalence of markers of hepatitis viruses A, $\mathrm{B}, \mathrm{C}$ and of HIV in healthy individuals and patients of a Cambodian province. Southeast Asian J. Trop. Med. Public Health 24, 239-249.

Tournier-Lasserve, C., Audebaud, G., Brumpt, V., Jolly, M., Calvez, F., Mazaud, R., Imbert, X., Bazillio, R., 1970. Existence of a focus of human bilharziosis, in Cambodia in the Kratie area. I. Study of the 1st three clinical cases. Med. Trop. (Mars.) 30, 451-461 [in French].

Urbani, C., Sinoun, M., Socheat, D., Pholsena, K., Strandgaard, H., Odermatt, P., Hatz, C., 2002. Epidemiology and control of mekongi schistosomiasis. Acta Trop. 82, 157-168.

Vong, S., Perz, J.F., Sok, S., Som, S., Goldstein, S., Hutin, Y., Tulloch, J., 2005. Rapid assessment of injection practices in Cambodia, 2002. BMC Public Health 5, 56.

WHO, 2000. Ultrasound in Schistosomiasis. A Practical Guide to the Standardized Use of Ultrasonography for the Assessment of Schistosomiasis-related Morbidity. Report of the TDR Second International Workshop, Niamey, Niger. World Health Organization, Geneva.

WHO, 2007. Ultrasound in Asian schistosomiasis: a practical guide to the standardized use of ultrasonography for the assessement of morbidity due to S. japonicum and S. mekongi, established at the Third Regional Network Meeting on Asian Schistosomiasis (RNAS) in May, 2002, Phnom Penh, Cambodia, and in April 2005 in Yueyang, Hunan Province, China. Draft meeting report. 